\title{
Naïve Realism and the Conception of Hallucination as Non-Sensory Phenomena
}

\author{
Takuya Niikawa \\ Chiba University and Fuji Women's University \\ BIBLID [0873-626X (2017) 46; pp. 353-381] \\ DOI: $10.1515 /$ disp-2017-0010
}

\begin{abstract}
In defence of naïve realism, Fish has advocated an eliminativist view of hallucination, according to which hallucinations lack visual phenomenology. Logue, and Dokic and Martin, respectively, have developed the eliminativist view in different manners. Logue claims that hallucination is a non-phenomenal, perceptual representational state. Dokic and Martin maintain that hallucinations consist in the confusion of monitoring mechanisms, which generates an affective feeling in the hallucinating subject. This paper aims to critically examine these views of hallucination. By doing so, I shall point out what theoretical requirements are imposed on naïve realists who characterize hallucinations as non-visual-sensory phenomena.
\end{abstract}

\section{Keywords}

Naïverealism, hallucination, introspection, visual experience, disjunctivism.

\section{Naïve realism and hallucination}

This paper aims to discuss the tenability of the eliminativist view of hallucination, according to which hallucinations lack visual phenomenology. In order to do this, I shall critically examine Heather Logue's austere eliminativist view of hallucination (Logue 2012a) and Jérôme Dokic and Jean-Rémy Martin's moderate eliminativist view of hallucination (Dokic and Martin 2012). In this section, I set out the background to a related discussion about naïve realism and hallucination. Against this background, I will briefly introduce Logue's and Dokic and Martin's views. 
What I refer to as naïve realism is the view that the phenomenology of veridical visual experience is constituted by the subject's seeing an environmental object with visible properties, rather than representing an external scene or sensing a private mental object. ${ }^{1,2}$ It has been argued that naïve realism can provide the best account of demonstrative thoughts about environmental objects (Campbell 2002; Raleigh 2011), and that naïve realism has an epistemological advantage over other views (Johnston 2006, 2011; Logue 2012b). This paper assumes that naïve realism is motivated by these semantic and epistemological arguments. ${ }^{3}$

Importantly, naïve realism does not state anything about hallucinations. What should naïve realists say about the phenomenology of hallucination? Although there are various types of hallucinations, this paper focuses on total and neurally matching hallucinations. This is because total and neurally matching hallucinations offer the most difficult challenge to naïve realism, namely, the screening off problem (I will discuss this problem later). A total hallucination is such that the hallucinating subject is not seeing any environmental object. A neurally matching hallucination is a hallucination such that there is a nomologically possible world in which the subject with the same brain states/activities veridically perceives an environmental object. ${ }^{4}$

${ }^{1}$ This does not mean that the phenomenology of veridical visual experience is wholly determined by the objects and their visible properties that the subject is seeing. The manner of seeing may also be a determining factor. For a relevant discussion, see French 2014.

${ }^{2}$ Seeing is relational in the sense that a subject can see an object only if the object exists. Seeing is different in this respect from visually representing, which is not relational. I, however, leave open the question of how to understand the nature of seeing. Following Logue (2012a), I assume that naïve realism does not rule out the possibility that seeing is partially constituted by the subject's representational states.

${ }^{3}$ For other advantages of naïve realism, see Fish 2009 (sec. 3.6), Kennedy 2009 and Martin 2002.

${ }^{4}$ Although this sort of hallucination is usually called "causally matching hallucination", I prefer "neurally matching" to "causally matching". This is because the term "causally matching" seems to imply that a visual experience is something caused by brain states/activities, but naïve realists may want to reject this implication. For instance, naïve realists may want to say that visual experience is not 
If a hallucination of a red apple is neurally matching, the hallucinatory experience is introspectively indiscriminable from a veridical visual experience that the subject would have if she veridically saw a red apple with the same brain states/activities, in the sense that the hallucinating subject cannot tell by introspection alone that the hallucinatory experience does not have the property of being a veridical visual experience of the sort. ${ }^{5}$ By "hallucination" and "hallucinatory experience" I mean those of the total and neurally matching kind.

Let us turn to the question mentioned above: what should naive realists say about the phenomenology of hallucination? The first choice faced by naïve realists is whether or not they should accept the idea that hallucinations have visual phenomenology. If naïve realists accepted this in accordance with common sense, they would be required to specify the metaphysical nature of the visual phenomenology of hallucination as being distinct from that of the visual phenomenology of veridical experience.

There are many possible positive accounts of the phenomenology of hallucination. For instance, naive realists may state that the phenomenology of hallucinatory experience is constituted by the subject's representing a scene where there is actually no such scene that is perceptually related to the subject. Alternatively, naïve realists may state that the phenomenology of hallucination is constituted by the subject's sensing a private mental item. Naïve realists who give such positive accounts of the phenomenology of hallucination are typically called "positive disjunctivists".

Martin (2004: 70-71) attacks positive disjunctivism by presenting the "screening off argument". The argument can be delineated as follows. Let us assume that the visual phenomenology of hallucination consists in the subject's having a certain property $P$. Since the external cause of a hallucination does not constitutively contribute to its phenomenology (a hallucination can, in principle, occur even without any external cause, i.e., purely by means of the brain's

caused by, but is rather grounded in certain brain states/activities. The term "neurally matching" seems more appropriate in this respect.

${ }^{5}$ It is typical to understand the introspective indiscriminability between veridical and hallucinatory experiences in this manner. See Fish 2009: 86-88 and Soteriou 2016: 220. 
spontaneous activities), $P$ supervenes on the brain states of the hallucinating subject. Since $P$ supervenes on the brain states of the hallucinating subject, the corresponding perceiving subject also has $P$. Since having $P$ is sufficient for the subject to have an experience with visual phenomenology, it is reasonable to think that the phenomenology of veridical visual experience also consists in the subject's having $P$. This suggests that having $P$ screens off the seeing of environmental object itself from the explanation of the phenomenology of veridical visual experience. If seeing is explanatorily redundant in this way, then naïve realism collapses or, at least, loses its theoretical attractiveness. ${ }^{6}$

To avoid the screening off problem, Martin $(2004,2006)$ endorses negative disjunctivism, which states that the phenomenology of hallucination is exhaustively captured by saying that it is introspectively indiscriminable from a veridical visual experience and that there is no further account of the phenomenology of hallucination. As is well known, however, there are many objections to Martin's negative disjunctivism. ${ }^{7}$ If the only way to defend naïve realism from the screening off problem is to adopt negative disjunctivism, then those who are persuaded by such objections would deem naïve realism untenable.

In order to save naïve realism from this predicament, Fish (2008, 2009: chap. 4) develops a cognitive view of hallucination. According to this view, hallucination is defined as a mental state/event which has the same cognitive effects (judgements, behaviours and so on) as certain kinds of veridical perception, without the subject actually seeing anything relevant. In arguing for the cognitive view of hallucination, Fish (2009: 93) accepts the eliminativist position that hallucinations lack visual phenomenology. Because of this eliminativist commitment, Fish can eschew the screening off problem. If hallucinations do not have visual phenomenology, then there is no explanation called for. It follows from this that there is nothing that can screen off seeing from explaining the phenomenology of veridical

\footnotetext{
${ }^{6}$ There are some attempts to show that the screening off argument is unsound, which I do not discuss here (Johnston 2004; Pautz 2010; Conduct 2012; Hellie 2013).

${ }^{7}$ For these objections, see Smith 2008, Conduct 2011, Logue 2010, 2012a and Zimmerman 2012.
} 
visual experience.

However, the cognitive view of hallucination runs into some other problems. I will describe one of them, which I think is the most crucial. ${ }^{8}$ When a subject is hallucinating a red apple without any reason to suspect that it is hallucinatory, the subject typically comes to believe that there is a red apple before her and that she herself has a visual experience with red-apple-phenomenology. This suggests that a hallucination rationally makes its subject inclined to form certain perceptual and introspective beliefs, where $\mathrm{X}$ rationally makes $\mathrm{S}$ inclined to believe $\mathrm{P}$ if and only if (1) X makes $\mathrm{S}$ inclined to believe $\mathrm{P}$ and (2) X makes it reasonable for $\mathrm{S}$ to believe P. (In saying that a subject is rationally inclined to believe $\mathrm{P}$, I mean that a subject is inclined to believe $\mathrm{P}$ and it is reasonable for $\mathrm{S}$ to believe P.) Arguably, one mission of the philosophical theory of hallucination is to (at least partially) explain why a hallucination rationally makes its subject inclined to form certain perceptual and introspective beliefs. However, the cognitive view provides no answer to this question. According to the cognitive view, hallucination is characterized as a mental state/event that produces particular cognitive effects including such beliefs. The claim that hallucination is a mental state that produces the perceptual belief that there is an apple before the subject and the introspective belief that the subject has a visual experience with certain phenomenology does not imply anything as to why the hallucination rationally makes its subject inclined to form these beliefs. ${ }^{9}$ Thus, the cognitive view of hallucination does not explain why a hallucination rationally makes its subject inclined to form certain perceptual and introspective beliefs.

This problem shows that Fish's positive characterization of hallucination is inadequate, but this does not mean that eliminativism itself is wrong. Eliminativists may be able to account for the rational force of hallucination by giving some other positive characterizations of hallucination. Given that eliminativists do not face the screening

\footnotetext{
${ }^{8}$ I partially owe this idea to Pautz (2013: sec. 2), where he presents the explanatory argument and the justification argument against Fish.

${ }^{9}$ As for other problems with Fish's cognitive view, see Logue 2012a, Dokic and Martin 2012 and Martin 2013. Although he replies to some of these objections in Fish 2013, his reply does not seem to be comprehensive.
} 
off problem, it is worth examining the tenability of other sorts of eliminativist theories of hallucination.

Logue (2012a) develops an eliminativist view of hallucination, according to which hallucinations are non-phenomenal, perceptual representational states. To the best of my knowledge, no explicit objection has been directed to this view. In Section 2 and 3, I will carefully examine Logue's eliminativist view and argue against it. Dokic and Martin (2012) are also committed to the eliminativist position that hallucinations lack visual phenomenology, claiming that hallucinations consist in the confusion of monitoring mechanisms. Little attention has been directed to this view. In Section 4, I will critically examine Dokic and Martin's view of hallucination. As the result of the discussions in Sections 2-4, we will be able to see what theoretical requirements are imposed on naive realists who deny that hallucinations have visual phenomenology.

\section{Logue's eliminativist view of hallucination}

This section aims to introduce the eliminativist view of hallucination that Logue (2012a) has developed, focusing on its explanatory potential. It is important to first note that Logue adopts an austere sort of eliminativist view, which states not only that hallucination lacks visual phenomenology but that it lacks any sort of phenomenology (Logue 2012a: 182). On this view, hallucinations are not even phenomenal states, let alone visual sensory experiences. Why does Logue need to commit herself to such an extreme view? If you only want to avoid the screening off problem, it seems sufficient to say that hallucinations lack visual phenomenology, and this is compatible with the claim that hallucinations have some non-visual sort of phenomenology. Even if hallucination has non-visual phenomenology and the latter is explained by a property $P$ that supervenes on the subject's brain state, there is no reason to think that $P$ can also explain the visual phenomenology of veridical perception.

The reason why Logue adopts the austere eliminativist view is to avoid the double phenomenology problem, which is delineated as follows: If a hallucination involves some phenomenology, it should supervene on the brain state of its subject. From this, it follows that a perceiving subject with the same brain state also undergoes the same sort 
of phenomenology. Then, however, this perceiving subject should have two sorts of phenomenology in its experience, normal visual phenomenology and the non-visual phenomenology shared with the hallucinating subject. This seems phenomenologically implausible. Reflecting on our veridical visual experience, it does not seem that we undergo double phenomenology, i.e. visual phenomenology and some other sort of phenomenology. All that we undergo in the veridical case would be visual phenomenology. Hence, any view from which it follows that a veridical perceptual experience has some sort of phenomenology in addition to visual phenomenology is implausible. This is the double phenomenology problem.

It may seem that the austere eliminativist view is implausible or even ridiculous. As many opponents of naïve realism, such as Chalmers (2006: 53, n1) and Pautz (2010: 236), simply assume that hallucinations are phenomenal, the austere eliminativist view is typically not argued against but merely ignored. Nevertheless, it clearly begs the question against the austere eliminativist to simply say that hallucinations must be phenomenal. Are there any convincing non-question-begging arguments against the austere eliminativist view?

The first apparent problem faced by the austere eliminativist view is similar to the one with Fish's cognitive view of hallucination. Suppose that a subject is hallucinating a red apple. The philosophical theory of hallucination should be able to (at least in part) explain why the hallucination rationally makes its subject inclined to believe that there is a red apple before her and that she herself has a visual experience with red-apple-phenomenology. If we allow the hallucinatory experience to have red-apple-phenomenology - a natural description of which is that a red apple is presented in her visual field-, then by appealing to the red-apple-phenomenology, we are able to explain why the hallucination rationally makes the subject inclined to form those perceptual and introspective beliefs. However, this explanation is not available to the austere eliminativist view, since it denies that hallucinations have visual phenomenology. Then, how can the austere eliminativist view explain why a hallucination rationally makes its subject inclined to form certain perceptual and introspective beliefs?

Logue endorses a representationalist version of the austere eliminativist view (henceforth, RE), which states that "total hallucinations 
fundamentally consist in the subject perceptually representing her environment as being a certain way, but they lack phenomenal character" (2012a: 183). On this view, the hallucination of a red apple has visual representational content involving a red apple, despite the fact that the content is not associated with phenomenology. RE seems able to provide a plausible explanation as to why a hallucination rationally makes its subject inclined to form a perceptual belief. The explanation is as follows: the hallucinating subject is rationally inclined to believe that there is a red apple before her because she perceptually represents a red apple as being before her. However, this explanation does not directly apply to the introspective belief that the subject herself has a visual experience with red-apple-phenomenology. The rational inclination to form the introspective belief is not adequately explained by simply saying that the hallucinating subject perceptually represents a red apple as being before her, since RE does not allow that the representational content is associated with red-apple-phenomenology. What explanation can RE give for the rational inclination to form the introspective belief?

In order to explain why a hallucinating subject is rationally inclined to form an introspective belief, Logue appeals to the introspective indiscriminability of a hallucination from a corresponding veridical perception. According to Logue, a particular hallucination is introspectively indiscriminable from a veridical experience of a certain kind if and only if the hallucination is "such that the subject cannot tell that it doesn't have the property of being a veridical experience of that kind (e.g., the property of being a veridical experience of a yellow, crescent-shaped thing)" (2012a: 176). In light of this analysis, Logue claims that "a subject of a hallucination as of a yellow, crescent-shaped thing cannot know by introspection alone that her experience doesn't have the property of being a veridical experience of such a thing, and is thereby inclined to believe that her state has the same phenomenal character as such a veridical experience" (2012a: 183-184). Following this idea, RE seems able to explain why a hallucination rationally makes its subject inclined to believe that she herself has a visual experience with red-apple-phenomenology by saying that this is because she cannot know by introspection alone that her hallucinatory experience lacks the property of being a veridical visual experience of a red apple. 
As Logue noticed, however, this leads to another explanatory task: austere eliminativists "need to explain how a hallucination could be subjectively indiscriminable from a certain kind of veridical experience when they differ so radically" (2012a: 184). One may think this is the most challenging task for RE. How is it possible that a non-phenomenal state be introspectively indiscriminable from a phenomenal experience? Logue answers this question by appealing to the sharing of introspectible representational content by a hallucination and a corresponding veridical perception. Logue claims that veridical perception and hallucination have in common the property of perceptual representing, and that "the content of the representational state is introspectively accessible by the subject" (Logue 2012a: 185). If a hallucinating subject is in the same introspectible representational state as that of a perceiving subject (and the hallucination does not have any other introspectively detectable feature showing that it is not a visual phenomenal experience), the hallucinating subject cannot know by introspection alone that her experience lacks the property of being a veridical phenomenal experience. In this way, RE can explain how a hallucination can be introspectively indiscriminable from a corresponding veridical visual experience even though they differ radically.

We have so far seen that RE seems able to offer an explanation for why a hallucination rationally makes its subject inclined to form perceptual and introspective beliefs. Likewise, RE seems able to explain how a hallucination as a non-phenomenal state can be introspectively indiscriminable from a corresponding veridical visual experience. Considering this, it is at least fair to say that RE is not so easily refutable as it might appear to be at first sight. RE is thus worth taking seriously as a potential naïve realist account of hallucination. ${ }^{10}$

\section{Objections to Logue's RE}

In this section, I will give two objections to RE. The first objection focuses on the causal roles of visual phenomenology. The second

${ }^{10} \mathrm{RE}$ allows that cognitively unimpaired subjects can make a radical introspective mistake about the presence or absence of visual phenomenology. You may suspect that it is implausible. Logue (2012a: sec. 5) addresses this worry in detail. 
objection casts doubt on the idea that we can introspectively access some representational content of hallucination that is not associated with phenomenology.

\subsection{First objection: higher order screening off worry}

One worry we may have about RE is that it seems to give rise to the implausible consequence that visual phenomenology is cognitively and rationally impotent or useless. When hallucinating a red apple, the subject is rationally inclined to believe that there is a red apple before her. According to RE, this can be explained in terms of the subject's perceptually representing an apple as being before her. With this in mind, consider the veridical case in which an internal twin of the hallucinating subject is successfully seeing a red apple. The perceiving subject is also rationally inclined to believe that there is a red apple before her. According to Logue, perceptually representing "is simply a constituent of" seeing (2012a: 180). From this, it would follow that the perceiving subject is also perceptually representing a red apple as being before her. It thus seems that the rational inclination to form a perceptual belief can also be fully explained in terms of the subject's representing a red apple as being before her without reference to the phenomenology of the veridical visual experience that she is undergoing. This suggests that the phenomenology of veridical visual experience does not play any role in explaining why the subject is rationally inclined to form the perceptual belief. However, this consequence seems implausible. Reflecting on my current visual experience of a laptop, it seems that I am rationally inclined to believe that there is a laptop before me because of the visual phenomenology of the experience, the natural description of which is that a laptop is presented in my visual field. ${ }^{11}$

\footnotetext{
${ }^{11}$ Some representationalists may want to say that the cognitive features of hallucination are explained in terms of the representational content of hallucination. Is this compatible with the first-personal impression that I am rationally inclined to believe that there is a laptop before me because of the visual phenomenology of my current experience? The answer is in the affirmative. A standard representationalist states that the visual phenomenology supervenes on (or is identical to) the representational content of the experience. Given this, the standard representationalist can reasonably claim that the rational inclination in question is fundamen-
} 
This objection to RE is similar in structure to the screening off argument, but it concerns the role of visual phenomenology in explaining a cognitive and rational feature of veridical visual experience, rather than the role of seeing in explaining visual phenomenology. Thus, I call this argument the "higher order screening off argument".

Logue might simply swallow the apparently counterintuitive consequence of RE that the visual phenomenology has nothing to do with the subject's inclination to form a perceptual belief and accord some other roles to it. In a different paper (Logue 2012b), Logue argues that the motivation for naïve realism is that only naive realism enables us to acquire a special sort of knowledge about instances of shape properties through the phenomenology of veridical visual experience. Based on such consideration, Logue may claim that visual phenomenology is not cognitively useless because it serves to explain how we acquire this sort of knowledge, though it does not serve to explain other cognitive and rational features of hallucination, such as its ability to make the subject rationally inclined to form certain perceptual beliefs.

Perhaps, it might be acceptable to say that the visual phenomenology does not play a role in explaining why a veridical visual experience rationally makes its subject inclined to form a perceptual belief. (Although this would be controversial, I do not further discuss this issue in this paper.) However, the same cannot be said in regards to its ability to generate the rational inclination to form an introspective belief. When hallucinating a red apple, the subject is also rationally inclined to believe that she herself has a visual experience with red-apple-phenomenology. RE explains this by saying that the hallucination is introspectively indiscriminable from a veridical visual experience of a red apple, and it accounts for this introspective indiscriminability by saying that the hallucinating subject is in the same introspectible perceptual representational state as the subject who is actually seeing a red apple. It may seem that this two-step explanation leads to the claim that being in a perceptual representational state whose content involves a red apple is sufficient for having the inclination to form the introspective belief that she herself has a visual experience with red-apple-phenomenology. Note that there 
is no reference to visual phenomenology in this description of the sufficient condition for having the inclination to form the introspective belief and that a corresponding perceiving subject also satisfies this sufficient condition. This suggests that visual phenomenology does not play any role in explaining why a veridical visual experience rationally makes its subject inclined to form the introspective belief. Generalizing this, visual phenomenology does not play any role in explaining why a visual experience rationally makes its subject inclined to form an introspective belief about visual phenomenology.

This is an unacceptable consequence. In fact, Logue admits that it is too bitter to swallow, but she argues that RE does not need to accept it. Logue claims:

Being in a perceptual representational state] isn't sufficient to explain why [the hallucinating subject] is inclined to believe that she's having an experience with perceptual phenomenal character-another crucial part of the explanation is that the representational state is normally a constituent of a state that does have perceptual phenomenal character. The explanatory power of the representational state depends upon it normally being a part of [a veridical visual experience with visual phenomenology. (2012a: 187)

This means that the reference to the visual phenomenology of veridical experience is indispensable to explain why a hallucination, as a non-phenomenal state, rationally makes the subject inclined to believe that she herself has an experience with visual phenomenology. Thus, RE allows visual phenomenology to serve to explain the rational inclination to form an introspective belief about visual phenomenology.

There is, however, a further objection to RE to which Logue's defence of RE does not apply. When we ask for a psychological explanation as to why a hallucinating subject is rationally inclined to believe that she herself has a visual experience with red-applephenomenology, we are at least in part concerned with what mental state/event causes or grounds the inclination to form the introspective belief. Advocates of RE are likely to answer this question by saying that the inclination in question is caused by (or grounded in) the subject's perceptual representational state whose content involves a red apple, with certain background mental conditions. Since the 
perceptual representational state is a part of a veridical visual experience of a red apple, it is shared by the subject who is actually seeing a red apple. Likewise, there is no reason to deny that the same background mental conditions hold in the perceiving subject. Hence, when a subject is seeing a red apple and thereby rationally inclined to believe that she herself has a visual experience with red-applephenomenology, it is reasonable to think that the inclination is also caused by (or grounded in) the perceptual representational state with the background mental conditions, which are all shared by the hallucinating subject. In this causal (or grounding) explanation, there is no reference to the phenomenology of the veridical visual experience of a red apple, namely, the red-apple-phenomenology. This suggests that the red-apple-phenomenology does not contribute to causing (or grounding) the inclination to form the introspective belief that she herself has a visual experience with red-apple-phenomenology. This leads to the consequence that even in a case in which a subject is actually undergoing an experience with certain visual phenomenology, the inclination to form an introspective belief about the visual phenomenology is not (even partially) caused by (or grounded in) the visual phenomenology itself. It is this consequence that I find implausible.

One might object to a hidden assumption in the above argument that there cannot be causal (or grounding) overdetermination about the inclination to form certain introspective beliefs, stating that the inclination can be doubly caused by (or grounded in) a perceptual representational state and certain visual phenomenology. ${ }^{12}$ However, this objection does not work; there is reason to think that RE cannot even admit this sort of overdetermination.

My claim is that if (I) a perceptual representational state is a constituent of the phenomenology of veridical visual experience and (II) the perceptual representational state can cause (or ground) the inclination to form certain introspective beliefs, then the phenomenology of veridical visual experience cannot be appropriately counted as a cause (or ground) of the inclination. Since advocates of RE clearly accept (II), in what follows I will first demonstrate that RE

\footnotetext{
${ }^{12}$ Even if this sort of overdetermination is permissible, it would at least be a theoretical burden.
} 
is committed to (I) and then argue for the conditional claim itself.

As we have seen, Logue (2012a: 180) claims that a perceptual representational state "is simply a constituent of" seeing. Naïve realism states that the phenomenology of veridical visual experience is constituted by the subject's seeing an environmental object with visible properties. Note that naive realists must deny that seeing is wholly constituted by a perceptual representational state; otherwise, naïve realism would collapse into some sort of representationalism, which states that the phenomenology of veridical visual experience is wholly constituted by the subject's visually representing. ${ }^{13}$ Hence, naïve realists must claim that seeing has an additional constituent other than a perceptual representational state.

The additional constituent would be a certain causal-informational chain between a perceptual representational system and an environmental object, where a perceptual representational state is caused by the event that is located at the beginning of the chain. ${ }^{14}$ That is to say, seeing an object is constituted by a perceptual representational state and a causal-informational chain between a perceptual representational system and the object. Given the transitivity of constitution, $\mathrm{RE}$ is committed to the claim that the phenomenology of veridical visual experience is constituted by a perceptual representational state and the causal-informational chain from an environmental object up to a perceptual representational system.

For convenience, let us use $R S$ to indicate a perceptual representational state, $C I C$ to indicate the causal-informational chain from an environmental object up to a perceptual representational system, $V P$ to indicate a veridical visual experience with visual phenomenology and $I B$ to indicate the inclination to form certain introspective beliefs

\footnotetext{
${ }^{13}$ I do not claim that all naïve realists allow seeing to be in part constituted by a perceptual representational state. Some of them may contend that seeing is irreducible in the sense that there is no more basic constituent of seeing. What is more, some representationalists may agree that seeing is constituted by a perceptual representational state and some other additional constituent, while contending that the phenomenology of veridical visual experience is wholly constituted by the perceptual representational state.

${ }^{14}$ I leave open how we should flesh out the causal-informational chain in question. For this task, we can appeal to cognitive psychological literature (for example, Palmer 1999).
} 
about visual phenomenology. Given this abbreviation, the above conditional claim is formulated as follows: if RS is a constituent of VP and RS can cause (or ground) IB, then VP cannot be appropriately counted as a cause (or ground) of IB. (From the argument in the previous paragraph, it follows that VP is constituted by RS and CIC.) In the rest of this subsection, I will argue for the conditional claim.

Let us first note the following three presuppositions: (1) RS can cause (or ground) IB with background mental conditions without CIC, (2) any event that is located in CIC cannot directly cause (or ground) $\mathrm{IB}^{15}$ and (3) the causal (or grounding) potential of RS does not disappear when it becomes a constituent of VP. There is no good reason for Logue to deny any of these presuppositions. With this in mind, let us consider a case in which $R S, C I C$ and VP occur and thereby $I B$ occurs. In this case, no symmetric causal overdetermination of IB by RS and VP occurs. The overdetermination in question occurs only if the following counterfactual holds: if RS had not occurred, VP would have caused IB (Céspedes 2016: 4). However, this counterfactual does not hold, since if RS had not occurred, VP would not have occurred, since VP is partially constituted by RS. Furthermore, any relevant sort of asymmetrical causal overdetermination does not hold. IB is asymmetrically causally overdetermined by RS and VP if (1) VP (RS) causes IB, (2) RS (VP) does not cause IB and (3) if VP (RS) had not occurred, RS (VP) would have caused IB (Céspedes 2016: 6). However, the second condition — RS does not cause IB - is inconsistent with the presupposition that the causal potential of RS does not disappear when it becomes a constituent of VP. In the case of the other direction, the corresponding third condition- - RS had not occurred, VP would have caused IB - cannot hold for the same reason as in the case of symmetric causal overdetermination. The same can apply to the grounding overdetermination. ${ }^{16}$

Provided that we know that RS can cause (or ground) IB with background mental conditions, is there any sense other than the

\footnotetext{
${ }^{15}$ Here I plausibly assume that the event that is located at the beginning of CIC cannot cause or ground IB without causing RS.

${ }^{16}$ It may be controversial whether asymmetric overdetermination can, in principle, holds for grounding relation (Bernstein 2016). If asymmetric grounding overdetermination is impossible, it is a positive support for my argument.
} 
one of overdetermination in which it is appropriate to say that VP causes (or grounds) IB? Remember that VP is constituted by RS and $\mathrm{CIC}$, where RS is caused by the event that is located at the beginning of CIC. If the presence of CIC makes a difference to the manner in which IB is caused by (or grounded in) RS in comparison with the case in which IB is caused by (or grounded in) RS without CIC, then there may be a context in which it is more appropriate to say that IB is caused by (or grounded in) VP than to say that IB is caused by (or grounded in) RS. ${ }^{17,18,19}$ Given that any event that is located in CIC cannot directly cause IB, there seems to be only two possible ways for CIC to make a difference to the manner in which IB is caused by RS. The first is that the presence of CIC alters the background mental conditions against which IB is caused by (or grounded in) RS. The second is that CIC directly influences the process through which RS causes IB with the help of background mental conditions. ${ }^{20}$ (I do not think there is something like "grounding process" analogues to the causal process at issue, since grounding is synchronic.) In what follows, I will argue that neither occur.

Let us first remember that we are dealing with the total and neurally matching hallucinatory case in which there is no internal difference between hallucinating and (corresponding) veridically perceiving subjects. This means that the presence of CIC, which marks the veridicality of the visual experience, does not make any difference to the subject's relevant mental conditions in comparison with the case in which CIC is absent, namely, a hallucinatory case. It follows from this that the presence of CIC does not make any difference to the background mental conditions in question.

Second, let us remember that the event that is located at the beginning of CIC is a causal precursor of RS. If an event $\mathrm{E}_{1}$ is a causal

\footnotetext{
${ }^{17}$ More concretely, this means that if the manner in which IB is caused by RS in a veridical case differs from the manner in which IB is caused by RS in a hallucinatory case because of the presence of CIC, it may be more appropriate to say that IB is caused by VP, which is constituted by RS and CIC, in the veridical case.

${ }^{18}$ For the context dependence of causal statements, see Reiss 2013.

${ }^{19}$ I appreciate a referee's suggestion to discuss this point.

${ }^{20}$ For the detail of causal processes, see Dowe 2008.
} 
precursor of $E_{2}$, then there is no temporal overlapping between $E_{1}$ and $E_{2}$. Thus, there is no temporal overlapping between CIC and RS. Given that $\mathrm{E}_{1}$ can directly influence a causal process between other two events $E_{2}$ and $E_{3}$ only when $E_{1}$ is temporarily (at least in part) overlapped with the causal process, it is implausible to think that CIC can directly influence the process through which RS causes IB.

We can thus conclude that the presence of CIC does not makes any difference to the manner in which IB is caused by (or grounded in) RS in comparison with the case in which IB is caused by (or grounded in) RS without CIC. It follows from this that there is no context in which it is less appropriate to say that RS causes (grounds) IB than to say that VP causes (or grounds) IB. This means that the phenomenology of veridical visual experience cannot be appropriately counted as a cause (or ground) of the inclination to form certain introspective beliefs about visual phenomenology.

Concluding this subsection, Logue fails to avoid the implausible consequence that even in a case in which a subject is actually undergoing an experience with certain visual phenomenology, the visual phenomenology cannot be appropriately counted as a cause (or ground) of the inclination to form an introspective belief about the visual phenomenology. This is a crucial problem with Logue's RE.

3.2 The second objection: introspective accessibility to nonphenomenal hallucination

Suppose that a subject is visually hallucinating a red apple. This hallucinatory experience is introspectively indiscriminable from a corresponding veridical experience; that is to say, the subject cannot know by introspection alone that the experience does not have the property of being a veridical experience of a red apple. Logue explains introspective indiscriminability by appealing to the sharing of introspectible representational content by the hallucinatory and veridical experiences. This explanation implies that the hallucinatory subject can introspectively know that the experience is about a red apple; in other words, it has visual representational content involving a red apple. From this, it follows that Logue's RE is committed to the the introspectability principle, that is: a hallucinating subject can introspectively know the representational content of hallucination. 
There is, however, first-personal reason to think that if hallucination lacks phenomenology, its representational content is not introspectible. Suppose that we are seeing a red apple and having a veridical visual experience with red-apple-phenomenology. We can introspect on this experience and can thereby know that it is about a red apple. However, if the experience suddenly loses its phenomenology, namely the red-apple-phenomenology, can we still introspectively know that it is about a red apple? Reflecting on my own visual experience of a red apple, the answer seems to be in the negative. It seems to me that the sudden loss of the red-apple-phenomenology leads to the loss of the introspective accessibility to the experience; I have no idea as to how I can introspectively know that it is about a red apple. The situation seems to be as if I suddenly become blind with respect to the red apple. This first-personal intuition is a case for the claim that if a veridical visual experience lacks phenomenology, the subject cannot introspectively know its representational content.

A similar consideration can apply to hallucinatory experiences. I can imagine a case in which I am hallucinating a red apple and having a hallucinatory experience with red-apple-phenomenology. It seems that I can introspect on this experience and can thereby know that it is about a red apple. However, it seems to me that if the experience suddenly loses its phenomenology, namely the redapple-phenomenology, I cannot introspectively know that it is about a red apple. This first-personal intuition is a case for the claim that if a hallucinatory experience lacks phenomenology, the subject cannot introspectively know its representational content. ${ }^{21,22}$

\footnotetext{
${ }^{21}$ This sort of first-personal consideration does not show that we cannot introspectively know the representational content of any non-phenomenal states. For instance, we seem able to introspectively know the representational contents of our beliefs, despite the fact that beliefs are not phenomenal (though it may be controversial whether beliefs are really non-phenomenal). Let me imagine that I am asked to answer whether I believe that there is an apple on my desk. It seems that I can somehow introspectively (correctly) judge whether I believe the proposition. Thus, the first-personal consideration only suggests that if a perceptual experience lack its phenomenology, their representational contents become introspectively inaccessible. I appreciate the referee's suggestion to make this clear.

${ }^{22}$ Objecting to this first-personal consideration, one may claim that the phenomenology of mental states does not play any role in their introspection, while
} 
In order to show that a hallucination as a non-phenomenal state is introspectible, advocates of RE need to explain how we can introspectively know its representational content. Logue presents an externalist theory of introspection, stating that "introspection of nonhallucinatory perceptual experience involves attending to things in one's environment (the objects of the experience), whereas introspection of total hallucination involves trying and failing to attend to such things" (2012a: 193). I do not discuss how much this theory succeeds in the case of non-hallucinatory perceptual experience; rather, I focus on whether it can explain how we can introspectively know the representational content of hallucination, which is not associated with phenomenology.

The question to be asked is, how can trying and failing to attend to environmental objects explain the introspective access to the representational content of hallucination? Although Logue does not explicitly say anything about it, we can plausibly assume that the "trying" part rather than the "failing" part is relevant. It is thus reasonable to think that Logue may say something like this: trying to attend to the object of a hallucinatory experience makes its representational content available to the hallucinating subject.

However, Logue's austere eliminativist commitment makes this explanation implausible. The question that should be asked here is, how is it possible to try to attend to the object of a hallucinatory experience in a case where it lacks visual phenomenology? If a subject is hallucinating a pink elephant and the hallucination has specific visual phenomenology - the description of which would be that something like a pink elephant is presented in the hallucinating subject's visual field - , then the subject can direct her attention to the visual area where the something is located to try to attend to the object of hallucination, namely the pink elephant. According to austere eliminativism, however, nothing is presented in a subject's visual field when the subject is hallucinating a pink elephant. In this case, how can the subject decide, in a non-arbitrary manner, in which direction

stating that introspection of mental states should be understood in terms of thirdpersonal cognitive psychology or some other scientific framework. This general claim needs independent justification, however, given that some philosophers argue that phenomenology plays an indispensable role for introspection (Smithies 2012; Silins 2012; Niikawa 2016). 
or to what visual area she should direct her attention in order to try to attend to the pink elephant? It seems impossible to try to attend to something in a non-arbitrary way if the subject does not know in advance in which direction or to what visual area she should direct her attention. Hence, Logue seems unable to explain how it is possible to decide, in a non-arbitrary way, in which direction or to what visual area a hallucinating subject should direct their attention in order to try to attend to the object of hallucination. Logue's externalist theory of introspection of hallucination seems incompatible with her commitment to austere eliminativism. ${ }^{23}$

My argument only aims at Logue's externalist theory of introspection of hallucination; it does not show that there is no theory of introspection of hallucination available to advocates of RE. As we have seen, however, we would have a first-personal intuition suggesting that if hallucination lacks phenomenology, it is not introspectible. Given this, the burden of proof would lie with advocates of RE. Unless they propose a particular theory of introspection that can account for the introspective access to the representational content of hallucination as non-phenomenal state, we can (temporarily) conclude that RE cannot secure the introspectability principle by relying on our first-personal intuition.

This argument shows that RE cannot secure the introspectability principle because of its austere eliminativist commitment. Hence, proponents of RE must discard either the austere eliminativist commitment or the introspectability principle. Note that RE seems able to account for the cognitive features of a hallucination, such as the indiscriminability from a veridical perception, by appealing to its introspectible representational content. Accordingly, proponents of RE

\footnotetext{
${ }^{23}$ Note that the dispute over the kinds of representational content of hallucination is irrelevant to the issue regarding the introspectibility of hallucination as non-phenomenal state. Regardless of whether the kind of representational content of hallucination is Russellian or Fregean, singular or general, or indexical or non-indexical, if hallucination is not phenomenal, we can reasonably ask how we can introspectively know the content of hallucination. Of course, it is questionbegging to merely assert that the representational content of hallucination is of the introspectible kind. Thus, it seems hopeless to try to explain why non-phenomenal representational content of hallucination is introspectible by appealing to the kind of the content.
} 
should preserve the introspectability principle. Hence, proponents of RE should retreat from the austere eliminativist view to the moderate eliminativist view, which states that hallucinations lack visual phenomenology but possess some other sort of phenomenology. Importantly, it is reasonable to think that if a representational state is phenomenal, it is introspectible. Thus, advocates of moderate eliminativism may be able to reasonably claim that the representational content of hallucination is introspectible.

Dokic and Martin (2012) offer a moderate eliminativist view, according to which hallucinations have a sort of affective phenomenology rather than visual sensory phenomenology. In the next section, I examine this moderate eliminativist view of hallucination as an alternative to Logue's RE.

\section{Metacognitive view of hallucination}

Dokic and Martin endorse a metacognitive view of hallucination, according to which "hallucinations are mere metacognitive projections, or phenomenal ghosts generated by 'confused' monitoring processes" (2012: 541). On this view, hallucinations lack visual phenomenology but possesses a feeling of reality (FoR), which is generated by a metacognitive confusion. They claim:

The hallucinating subject feels like she is perceptually open to the world itself. However, her feeling of reality results from a kind of MC [metacognitive] confusion, more precisely from the fact that low-level mechanisms have mistakenly tagged non-perceptual [hallucinatory] first-order processes as genuinely perceptual processes. (Dokic and Martin 2012: 538)

Although a FoR has been described here as a feeling of being "perceptually open to the world itself", it can also be described as a feeling of "belonging to the world" (Ratcliffe 2009) or "being perceptual confronted with a real thing or event" (Dokic and Martin 2012: 538).

In our context, it is important to note that FoR is in itself phenomenal. Assuming that the representational content of hallucination is reflected in the FoR, it is reasonable to think that the representational content is introspectible. Thus, the metacognitive view of hallucination seems compatible with the explanatorily advantageous 
view that hallucinations have introspectible representational content.

Nevertheless, we should not forget the reason why Logue is committed to the austere eliminativist view. It is to avoid the phenomenologically implausible consequence that veridical visual experience has double phenomenology. Can the metacognitive view avoid the double phenomenology problem? Suppose that a theory of hallucination states that hallucinations have a non-visual sort of phenomenology. There are two strategies to argue that the theory can avoid the double phenomenology problem. The first strategy is to explain why veridical visual experiences do not possess the non-visual sort of phenomenology. The second strategy is to argue that it is not phenomenologically implausible that a veridical visual experience has the non-visual sort of phenomenology in addition to visual sensory phenomenology. The metacognitive view can avoid the double phenomenology problem by using this second strategy. Reflecting on my current perceptual experience, I am also feeling like I am perceptually open to the world itself. It seems to me that my experience has this affective feeling in addition to visual sensory phenomenology. ${ }^{24}$ Thus, it is not phenomenologically implausible that a veridical visual experience has a FoR in addition to visual sensory phenomenology.

Can the metacognitive view explain cognitive features of a hallucination, such as the introspective indiscriminability from a veridical perception? According to Dokic and Martin, "what produces cognitive effects similar to the cognitive effects produced by a perception is the subject's feeling of reality, not the first-order processes themselves" (2012: 538). That is to say, the metacognitive view tries to explain cognitive features of hallucination by appealing to a FoR. Is this explanatory strategy hopeful? I doubt that it can succeed. In a nutshell, my worry is that a FoR cannot be contentful enough to explain the introspective indiscriminability of hallucinations from veridical perceptions. I will discuss this problem in the rest of this section. ${ }^{25}$

\footnotetext{
${ }^{24}$ Dokic and Martin (2017) discuss how FoR is integrated in veridical experiences in detail.

${ }^{25}$ Even if the metacognitive view can successfully explain cognitive features of hallucination, it may face something like the higher order screening off problem. This is because if cognitive features of hallucination are fully explained in
} 
Let us consider a hallucination of a red apple. The metacognitive view must be able to explain why the hallucination is introspectively indiscriminable from a veridical visual experience of a red apple. When a subject is veridically seeing a red apple, the subject has a unified experience in which visual sensory phenomenology and a feeling of reality are integrated. This complex phenomenology can be described as follows: the subject is feeling that she is perceptually open to a real red apple. Given this, the metacognitive view can explain the introspective indiscriminability in question only if it allows that the hallucinating subject is also feeling that she is perceptually open to a real red apple.

However, it is doubtful that a FoR in itself can have such a specific and determinate content which contains the reference to a red apple. It seems to be generally accepted that FoR as an affective feeling has at best unspecific and indeterminate content. This conception of FoR is supported by Dokic and Martin's account as to how a FoR is generated. They claim that FoR "result[s] from the operation of MP [metaperceptual] processes whose function is to monitor the quality of first-order processes, whatever contents are implicitly or explicitly processed" (2012: 540). This suggests that FoR correlates with not the content but the quality of first-order perceptual processes. The insensitivity to the content of the perceptual processes accounts for the fact that a FoR can only have unspecific and indeterminate content.

Dokic and Martin recognize this difficulty and suggest two possible solutions (2012: 541). First, they point to the possibility that the content of FoR can be enriched by cognitive penetration by beliefs and imaginings. Perhaps, the content of FoR can be enriched so as to become a bit more specific and determinate by some sort of cognitive penetration. However, it is doubtful that cognitive penetration can enrich the content of $\mathrm{FoR}$ to the extent that would be necessary for the content of FoR to become as specific and determinate as that of a visual sensory experience. Let us imagine that I am short-sighted and am seeing a sheep without my glasses. The experience would have unspecific and indeterminate content, the description of which would be that there is an obscure object before me. Even if I strongly believe that

terms of a FoR, then it seems that the same explanation holds for veridical perceptual cases. 
what I am seeing is a sheep, my visual experience remains obscure and indeterminate; it remains introspectively discriminable from the visual experience that I would have if I saw the sheep through my glasses. Likewise, even if I visually imagine a sheep, it does not radically change my visual experience. The gap in content between the obscure experience and the vivid experience of a sheep cannot be filled by cognitive penetration by beliefs and imaginings. I do not think that the degree of difference in content between FoR and visual sensory experience can be smaller than the degree of difference in content between the obscure visual experience and the vivid visual experience. Thus, the gap in content between FoR and visual sensory experience would not be filled by cognitive penetration by beliefs and imaginings. ${ }^{26}$

What is more, such cognitive penetration leads to a vicious circle. Let us consider again a case in which a subject is hallucinating an apple. According to the metacognitive view of hallucination, the subject is feeling like she is perceptually open to the world. If the content of this FoR can be enriched by beliefs such that the content involves an apple, the candidate belief would be that there is an apple before the subject, or that the subject has a visual experience with red-apple-phenomenology. However, the enriched FoR - the feeling that she is perceptually open to a real apple - is supposed to explain why the hallucinating subject is inclined to form these beliefs. The explanation for why a FoR inclines us to form certain beliefs should not involve the very beliefs whose formation the FoR is supposed to explain. This is clearly a vicious circle.

As for the second possible solution, Dokic and Martin (2012: 541) consider the possibility that a FoR brings about some visual sensation. If this visual sensation is sufficiently vivid and rich, advocates of the metacognitive view may be able to explain the introspective indiscriminability of a hallucination from a veridical perception in terms of the combination of a FoR and this visual sensation. However, if

\footnotetext{
${ }^{26}$ Dokic and Martin 2012: 541 refers to a patient with Parkinson disease as an example of a FoR having a specific and determinate content by cognitive penetration. However, this example does not show that the content of the patient's FoR is so specific and determinate that the patient cannot know by introspection alone that her experience does not have the property of being a veridical experience of a certain kind.
} 
visual sensation is something like two-dimensional visual impression, it is very doubtful that it can be vivid and rich enough to explain the introspective indiscriminability of a hallucination from a veridical perception. What could visual sensation be if it is not such a two-dimensional visual impression? Dokic and Martin do not explain what they mean by the term, let alone give any account of the nature of visual sensation. Unless an account of the nature of visual sensation is provided, the appeal to visual sensation is not helpful. Thus, it is fair to say that the two possible solutions that Dokic and Martin have mentioned are not appealing.

\section{Conclusion}

I have so far critically examined Logue's austere eliminativist view and Dokic and Martin's moderate eliminativist view of hallucinatory phenomenology. Here are the three main conclusions:

(1) The moderate eliminativist view is more hopeful than the austere eliminativist view, in that the former allows hallucinations to have some non-visual phenomenology and thereby some introspectible representational content.

(2) In order to explain the introspective indiscriminability of hallucinations from veridical perceptions, the moderate eliminativist view must allow that the representational content of hallucination, which is associated with its non-visual phenomenology, be significantly similar to the content of veridical experience, which is associated with its visual phenomenology.

(3) The moderate eliminativist must deal with the higher order screening off problem and the double phenomenology problem.

To construct a moderate eliminativist theory of hallucination, accordingly, naïve realists are required to account for the significant similarity between the content of hallucination and the content of veridical perception, each associated with a different kind of phenomenology, without being contradicted by the higher order screening off problem and the double phenomenology problem. This is clearly not an easy requirement to meet. The eliminativist view of 
hallucination, thus, is not an easy way out for naïve realists.

Let me close this paper by briefly sketching one way in which moderate eliminativists could answer this challenge. Naïve realists may attempt to meet this requirement by claiming that hallucinatory experiences involve imaginative phenomenology rather than perceptual phenomenology (Allen 2014). They might then explore to account for the significant similarity between the content of hallucination and veridical perception based on the similarity between the content of imaginative experience and perceptual experience (Allen 2014; Nanay 2016). At the same time, they could address the double phenomenology problem and the higher order screening off problem by appealing to the proposal made in neuroscience that visual imagination is based on an "off-line" use of the visual cognitive system, while visual perception is based on an "on-line" use of the same system, where the difference between the two modes is characterized in terms of the system's relation to the surrounding environment (Ganis, Thompson, and Kosslyn 2004). This suggests that the phenomenology of a (total and neurally matching) hallucinatory experience should supervene on the state of the visual cognitive system in the off-line mode. Given that the visual system cannot be used both on-line and off-line at the same time, it follows that a veridical experience with visual phenomenology based on the on-line use of the visual cognitive system cannot also have a hallucinatory phenomenology. Thus, the double phenomenology problem and the higher order screening off problem will not be a threat to this account. Although there are many tasks needed to fully defend this view, it seems that naïve realists adopting the moderate eliminativism should pursue this course. ${ }^{27}$

Takuya Niikawa

Chiba University

1-33, Yayoicho, Inage Ward, Chiba-shi, Chiba, 263-8522 Japan

\footnotetext{
${ }^{27}$ I would like to thank Katsunori Miyahara, Tricia Magalotti, Yasushi Ogusa, Yuya Shimizu for their detailed comments on this paper. I would also like to thank the members of POPS research group and the two anonymous referees for their helpful suggestions. I also thank Richard Stone and Yukika Ohmura for their careful proofreading. I gratefully acknowledge the generous support of the Japan Society for the Promotion of Science.
} 
Fuji Women's University Hanakawa Minami 4jo-5, Ishikari-shi, Hokkaido, 0613204, Japan niitaku11@gmail.com

\section{References}

Allen, Keith. 2014. Hallucination and imagination. Australasian Journal of Philosophy 93(2): 287-302.

Bernstein, Sara. 2016. Grounding is not causation. Philosophical Perspectives 30(1): 21-38.

Campbell, John. 2002. Reference and Consciousness. New York: Oxford University Press.

Céspedes, Esteban. 2016. Causal Overdetermination and Contextualism. Cham: Springer.

Chalmers, David J. 2006. Perception and the fall from Eden. In Perceptual Experience, ed. by Tamar S. Gendler and John Hawthorne, 49-125. New York: Oxford University Press.

Conduct, M. D. 2011. Naïve realism and extreme disjunctivism. Philosophical Explorations 13(3): 201-221.

Conduct, M. D. 2012. Naïve realism without disjunctivism about experience. Consciousness and Cognition 21(2): 727-736.

Dokic, Jérôme, and Martin, Jean-Rémy. 2012. Disjunctivism, hallucination and metacognition. WIREs Cognitive Science 3: 533-543.

Dokic, Jérôme. and Martin, Jean-Rémy. 2017. Felt reality and the opacity of perception. Topoi 36(2): 299-309.

Dowe, Phil. 2008. Causal processes. In The Stanford Encyclopedia of Philosophy, ed. by Edward N. Zalta, Fall 2008. Stanford: Metaphysics Research Lab, Stanford University. https://plato.stanford.edu/archives/fall2008/entries/ causation-process/.

Fish, William. 2008. Disjunctivism, indistinguishability, and the nature of hallucination. In Disjunctivism: Perception, Action, Knowledge, ed. by Adrian Haddock and Fiona Macpherson. New York: Oxford University Press.

Fish, William. 2009. Perception, Hallucination, and Illusion. Oxford: Oxford University Press.

Fish, William. 2013. Perception, Hallucination, and Illusion: reply to my critics. Philosophical Studies 163(1): 57-66.

French, Craig. 2014. Naive realist perspectives on seeing blurrily. Ratio 27(4): $393-413$

Ganis, Giorgio; Thompson, William L.; and Kosslyn, Stephen M. 2004. Brain areas underlying visual mental imagery and visual perception: an fMRI study. Brain Research. Cognitive Brain Research 20(2): 226-241.

Hellie, Benj. 2013. The multidisjunctive conception of hallucination. In Hallucination, ed. by Fiona Mapherson. Cambridge: MIT Press.

Johnston, Mark. 2004. The obscure object of hallucination. Philosophical Studies 
120(1-3): 113-183.

Johnston, Mark. 2006. Better than mere knowledge? The function of sensory awareness. In Perceptual Experience, ed. by T. S. Gendler and John Hawthorne. New York: Oxford University Press.

Johnston, Mark. 2011. On a neglected epistemic virtue. Philosophical Issues 21(1): $165-218$

Kennedy, Matthew. 2009. Heirs of nothing: the implications of transparency. Philosophy and Phenomenological Research 79(3): 574-604.

Logue, Heather. 2010. Getting acquainted with naïve realism: critical notice of Perception, Hallucination, and Illusion. Philosophical Books 51(1): 22-38.

Logue, Heather. 2012a. What should the naïve realist say about total hallucinations? Philosophical Perspectives 26(1): 173-199.

Logue, Heather. 2012b. Why naive realism? Proceedings of the Aristotelian Society 112(2pt2): 211-237.

Martin, Michael G. F. 2002. The transparency of experience. Mind and Language 4(4): 376-425.

Martin, Michael G. F. 2004. The limits of self-awareness. Philosophical Studies 120(1-3): 37-89.

Martin, Michael G. F. 2006. On being alienated. In Perceptual Experience, ed. by Tamar S. Gendler and John Hawthorne. New York: Oxford University Press.

Martin, Michael G. F. 2013. Shibboleth: some comments on William Fish's Perception, Hallucination and Illusion. Philosophical Studies 163(1): 37-48.

Nanay, Bence. 2016. Hallucination as mental imagery. Journal of Consciousness Studies 23(7-8): 65-81.

Niikawa, Takuya. 2016. Naïve realism and the explanatory role of visual phenomenology [Special Issue]. Argumenta-Journal of Analytic Philosophy 1(2): 219-231.

Palmer, Stephen E. 1999. Vision Science: Photons to Phenomenology. $1^{\text {st }}$ edition. Cambridge: The MIT Press.

Pautz, Adam. 2010. Why explain visual experience in terms of content? In Perceiving the World, ed. by Bence Nanay. New York: Oxford University Press.

Pautz, Adam . 2013. Do the benefits of naïve realism outweigh the costs? Comments on Fish, Perception, Hallucination and Illusion. Philosophical Studies 163(1): 25-36.

Raleigh, Thomas. 2011. Visual experience and demonstrative thought. Disputatio 4(30): 69-91.

Ratcliffe, Matthew. 2009. Belonging to the world through the feeling body. Philosophy, Psychiatry, and Psychology 16(2): 205-11.

Reiss, Julian. 2013. Contextualising causation part II. Philosophy Compass 8(11): $1076-1090$

Silins, Nicholas. 2012. Judgment as a guide to belief. In Introspection and Consciousness, ed.by Declan Smithies and Daniel Stoljar. New York: Oxford University Press. 
Smith, A. D. 2008. Disjunctivism and discriminability. In Disjunctivism: Perception, Action, Knowledge, ed. by Adrian Haddock and Fiona Macpherson, 181-204. New York: Oxford University Press.

Smithies, Declan. 2012. A simple theory of introspection. In Introspection and Consciousness, ed. by Declan Smithies and Daniel Stoljar. New York: Oxford University Press.

Soteriou, Matthew. 2016. Disjunctivism. New York: Routledge.

Zimmerman, Aaron. 2012. Introspection, explanation, and perceptual experience: resisting metaphysical. In Introspection and Consciousness, ed. by Declan Smithies and Daniel Stoljar. New York: Oxford University Press. 